\section{Kidney \\ Blood Pressure Research}

\title{
Brain Natriuretic Peptides in Atherosclerotic Renal Artery Stenosis and Effects of Renal Angioplasty
}

\author{
Elzbieta Nowakowska-Fortuna ${ }^{a}$ Aso Saeed ${ }^{a} \quad$ Gregor Guron ${ }^{a}$ Michael Fu \\ Ola Hammarsten ${ }^{b}$ Gert Jensen ${ }^{a}$ Hans Herlitz ${ }^{\mathrm{a}}$
}

aDepartment of Molecular and Clinical Medicine/Nephrology, Institute of Medicine, and 'Department of Clinical Chemistry and Transfusion Medicine, Institute of Biomedicine,

The Sahlgrenska Academy at the University of Gothenburg, Sweden.

\section{Key Words}

Brain natriuretic peptide $\bullet$ Renal angioplasty $\bullet$ Renal artery stenosis $\bullet$ Renovascular hypertension - Adiponectin

\begin{abstract}
Background: We hypothesized that plasma levels of brain natriuretic peptide (BNP) and $\mathrm{N}$-terminal pro-BNP (NT-proBNP) would be elevated, and adiponectin concentrations reduced, in patients with atherosclerotic renal artery stenosis (ARAS) and that BNPs might be used to identify patients who would benefit from percutaneous transluminal renal angioplasty (PTRA). Methods: Data were collected before renal angiography in 91 patients with hypertension and suspected ARAS (significant ARAS; $n=47$, and non-RAS; $n=44$ ) and in 20 healthy controls (C). In ARAS patients analyses were repeated four weeks after PTRA. Results: Ambulatory systolic blood pressure (ASBP) was significantly elevated in the ARAS group vs. both $C$ and non-RAS groups. Baseline plasma BNP and NT-proBNP levels were significantly elevated, and adiponectin concentrations reduced, in the ARAS group vs. $C$ but not vs. the non-RAS group. One month after PTRA, ASBP was reduced vs. baseline $(149 \pm 16$ to $139 \pm 15 \mathrm{~mm} p<0.01)$. Brain natriuretic peptides were not significantly affected by PTRA. Conclusions: Patients with ARAS showed elevated of BNP and NT-proBNP concentrations, and reduced levels of adiponectin, compared to healthy controls but not vs. hypertensive individuals without RAS. Our data do no support the use of BNP analyses in the identification of ARAS patients who will have a beneficial blood pressure response to PTRA.
\end{abstract}




\section{Kidney Blood Pressure Research}

Kidney Blood Press Res 2013;37:657-666

\begin{tabular}{l|l}
\hline DOI: $10.1159 / 000355746$ & (C) 2013 S. Karger AG, Basel
\end{tabular}

Published onlıne: December 15, 2013

www.karger.com/kbr

\section{Introduction}

Brain natriuretic peptide (BNP) and N-terminal pro-BNP (NT-proBNP) are secreted in equimolar amounts during hemodynamic and neurohormonal stress of the heart [1]. Because of their close correlation with the severity of symptoms and prognosis they have been developed as biomarkers in heart failure $[2,3]$. Brain natriuretic peptide has a serum half-life of $\approx 20$ minutes [4], while the corresponding value for NT-proBNP is $\approx 120$ minutes. Both BNP and NT-proBNP plasma concentrations increase in renal failure. However, elimination of NT-proBNP is likely more dependent on glomerular filtration than BNP, which is also cleared by receptor-mediated uptake and protease action, resulting in an increased NT-proBNP/BNP ratio in renal failure [5, 6]. The most important physiological actions of BNP take place in the kidney where it exerts natriuretic and diuretic effects, causes renal vasodilation, increases glomerular filtration rate and inhibits renin release [7]. In addition, in vitro data suggest that angiotensin II (Ang II) directly induces the synthesis and release of BNP [8]. Hence, plasma BNP may be increased in patients with renovascular hypertension at least partly due to an enhanced activity of the renin-angiotensin system [8-10].

The adipose tissue is not a passive energy depot, but in fact is an active endocrine organ secreting a variety of hormones, i.e. adipokines. Dysregulation of these adipokines is believed to be important for the development of cardiovascular disease in obesity [11]. Adiponectin is a 244 amino acid protein that is highly expressed by adipocytes and is present in human plasma [12]. Adiponectin has a variety of protective functions and is believed to exert antiinflammatory, anti-atherogenic and anti-diabetogenic effects [13]. As we previously have shown that patients with atherosclerotic renal artery stenosis (ARAS) have increased plasma levels of inflammatory biomarkers [14], and show an atherogenic lipoprotein profile [15], we speculated that ARAS patients may have reduced plasma levels of adiponectin that could contribute to the aforementioned abnormalities.

The aim of the present investigation was to examine plasma levels of BNPs and adiponectin in patients with ARAS. We hypothesized that brain natriuretic peptides would be elevated in ARAS patients and that higher levels might predict a favourable outcome of percutaneous transluminal renal angioplasty (PTRA).

\section{Subjects and Methods}

\section{Study participants}

Between 2003 and 2008, all patients at the Nephrology section, Sahlgrenska University Hospital, Gothenburg, Sweden, undergoing renal angiography for suspected renal artery stenosis (RAS) were considered for this study. Indications for angiography were hypertension (resistant, accelerated, malignant, or with elevation of serum creatinine during treatment with angiotensin converting enzyme (ACE) inhibitors or Ang II receptor blockers [ARBs]), hypertension accompanied by a progressive increase in serum creatinine levels, or recurrent pulmonary oedema without overt left ventricular dysfunction, together with a positive screening test for RAS by duplex ultrasonography or by CT- or MR-angiography ( $\geq 50 \%$ diameter stenosis). To avoid pharmacological interference with the renin-angiotensin-aldosterone system (RAAS), patients in whom treatment with ACE-inhibitors, ARBs or aldosterone receptor antagonists were clearly indicated (e.g. patients with congestive heart failure or diabetic nephropathy), were excluded. In remaining patients treated with RAAS inhibitors these agents were replaced by other antihypertensive drugs two weeks prior to renal angiography. Hence, included patients were not on any RAAS inhibiting drugs during the study period starting from two weeks prior to baseline measurements. In addition, only patients with unilateral ARAS were included and individuals with RAS of other aetiology, or with either bilateral RAS or stenosis of a solitary kidney, were excluded. Other exclusion criteria were renal size $<7.5 \mathrm{~cm}$ on the stenotic side, age $>80$ years, pregnancy or nursing, chronic kidney disease stage 5 (eGFR $<15 \mathrm{ml} / \mathrm{min}$ ), congestive heart failure, urinary albumin excretion $>1 \mathrm{~g} / 24 \mathrm{~h}$ (in diabetic patients $>0.3 \mathrm{~g} / 24 \mathrm{~h}$ ), contraindication for renal angiography/angioplasty e.g. serious contrast allergy, other forms of secondary hypertension, malignant 


\section{Kidney \\ Blood Pressure Research}

Kidney Blood Press Res 2013;37:657-666

\begin{tabular}{l|l}
\hline DOI: $10.1159 / 000355746$ & (C) 2013 S. Karger AG, Basel
\end{tabular}

Published onlıne: December 15, 2013

www.karger.com/kbr

659

disease or treatment with immune modulating drugs e.g. calcineurin inhibitors or oral steroids. Twenty age-matched healthy subjects without any medications were recruited from the database of the Gothenburg MONICA study and served as controls. The MONICA (multinational monitoring of trends and determinants in cardiovascular disease) project was established in the early 1980s to monitor trends in cardiovascular diseases and to relate these to risk factors [16]. The Ethics Committee of the University of Gothenburg approved the study and all participants gave written consent to participate.

\section{Protocol and measurements}

Ninety-one patients with hypertension and suspected RAS were included and all patients underwent renal angiography. Angioplasty was carried out on forty-seven patients with significant stenosis (ARASgroup). A significant RAS was defined as a lesion with a trans-stenotic mean arterial pressure gradient (MAPG) of $\geq 10 \mathrm{mmHg}$ or a $\geq 50 \%$ diameter stenosis on angiography in those cases in which the MAPG was not measured because of technical difficulties due to high-grade stenosis and luminal occlusion during the procedure. Forty-four individuals had no significant RAS and were therefore only subjected to diagnostic procedure (non-RAS group).

All patients were subjected to baseline measurements one day before angiography. Routine laboratory analyses, BNP, NT-proBNP and adiponectin were measured immediately before renal angiography in all patients. In patients that were subjected to PTRA analyses were repeated four weeks after intervention (except for adiponectin). Systolic and diastolic blood pressure (SBP and DBP) were measured after 5 minutes rest in the sitting position immediately before and one day and four weeks after renal angiography. Ambulatory (24h) SBP (ASBP) and DBP (ADBP) were measured by an ambulatory blood pressure system (Model 90217, Spacelabs Healthcare) one day before angiography and four weeks after PTRA. Estimated glomerular filtration rate (eGFR) was calculated according to the 4-variable equation from the Modification of Diet in Renal Disease (MDRD) Study. Notably, all patients with suspected RAS had been on treatment with a HMG-CoA reductase inhibitor (i.e. statin) for at least two weeks at the time of baseline measurements. The majority of patients was already on statin therapy at the time of study inclusion and continued with the same dosage throughout. In remaining patients treatment with simvastatin was started at the time of study inclusion in a daily dose of $20 \mathrm{mg}$ that was maintained throughout. In addition, most patients in both hypertensive groups were already on low-dose acetylsalicylic acid (ASA) at this time-point, while treatment with ASA was started 1-2 days before PTRA among those patients who were not already on this treatment. Treatments with statins and ASA were maintained unaltered in patients with ARAS throughout the study period. Healthy controls $(n=20)$ were only studied at one time-point and data were compared with baseline values from both hypertensive groups.

\section{Renal angiography and angioplasty}

Digital subtraction angiography was used for evaluating renal arteries. The procedures of renal angiography and PTRA have been described previously [17]. A 4 French catheter was used for measurements of intra-arterial pressure gradients. The diameter of stenosis was estimated manually in all cases. Indications for stent placement were angioplasty failure (elastic recoil or flow-limiting dissection resulting in $>30 \%$ residual luminal narrowing, absence of antegrade flow, or significant residual MAPG), or restenosis.

\section{Biochemical analyses}

Standard laboratory methods at the Department of Clinical Chemistry at Sahlgrenska University Hospital (SWEDAC approved according to European norm 45001) were used for routine analyses. Plasma renin activity (PRA) was measured by a radioimmunoassay (RIA) kit (DiaSorin, Stillwater, MN, USA), with inter-and intraassay coefficients of variation (CV) of less than 10\%. The plasma concentration of Ang II (Euro-Diagnostica, Malmö, Sweden) was also measured by RIA.

Brain natriuretic peptide was analysed using the manual Shionoria BNP method (CIS Bio international, Gif-sur-Yvette, France) on freshly thawed EDTA-plasma samples. The inter-sample CV was between 5.4 and 7.0 \%. The EDTA-plasma for BNP testing was frozen at $-70 \mathrm{C}$ within $2 \mathrm{~h}$ after collection. $\mathrm{N}$-terminal pro-BNP was analysed in serum with the Roche Elecsys system on Modular E 2551 with a CV between 3.7 and 5.0 $\%$. An ELISA kit from LINCO Research Inc. (St. Charles, MO, USA) was used for the quantification of plasma adiponectin (CV 8.55\%). 


\section{Kidney Blood Pressure Research}

\begin{tabular}{l}
\hline Kidney Blood Press Res 2013;37:657-666 \\
\hline \begin{tabular}{l|l} 
DOI: 10.1159/000355746 & (c) 2013 S. Karger AG, Basel \\
Published onlıne: December 15, 2013 & wwarger.com/kbr
\end{tabular} \\
\hline
\end{tabular}

Nowakowska-Fortuna/Saeed/Guron/Fu/Hammarsten/Jensen/Herlitz: Brain Natriuretic Peptides in Atherosclerotic Renal Artery Stenosis

Table 1. Baseline characteristics before renal angiography

\begin{tabular}{|c|c|c|c|c|}
\hline & $\begin{array}{l}\text { Healthy } \\
\text { controls } \\
(n=20)\end{array}$ & $\begin{array}{c}\text { Non-RAS } \\
(n=44)\end{array}$ & $\begin{array}{c}\text { ARAS } \\
(n=47)\end{array}$ & $\begin{array}{l}\mathrm{p} \text {-value } \\
\mathrm{K} \text {-W test }\end{array}$ \\
\hline Age (years) & $64 \pm 10$ & $61 \pm 11$ & $65 \pm 9$ & ns \\
\hline BMI $\left(\mathrm{kg} / \mathrm{m}^{2}\right)$ & $26 \pm 3$ & $27 \pm 5$ & $27 \pm 4$ & ns \\
\hline Gender (male \%) & $9(45 \%)$ & $23(52 \%)$ & $33(70 \%)$ & NA \\
\hline Current cigarette smoking, $\mathrm{n}(\%)$ & $2(10 \%)$ & $10(23 \%)$ & $14(30 \%)$ & NA \\
\hline Diabetes mellitus, n (\%) & - & $1(2 \%)$ & $9(19 \%) \dagger$ & NA \\
\hline Coronary artery disease, $\mathrm{n}(\%)$ & - & $14(32 \%)$ & $17(36 \%)$ & NA \\
\hline Atrial fibrillation, n (\%) & - & $3(7 \%)$ & $4(9 \%)$ & NA \\
\hline S-Triglyceride (mmol/L) & $1.4 \pm 0.6$ & $1.5 \pm 0.6$ & $1.8 \pm 0.8$ & ns \\
\hline S-LDL-C $(\mathrm{mmol} / \mathrm{L})$ & $3.0 \pm 0.9$ & $2.1 \pm 0.7^{*}$ & $2.5 \pm 0.8$ & $\mathrm{p}<0.01$ \\
\hline S-HDL-C (mmol/L) & $1.7 \pm 0.4$ & $1.4 \pm 0.4^{*}$ & $1.3 \pm 0.3^{*}$ & $\mathrm{p}<0.01$ \\
\hline
\end{tabular}

RAS, renal artery stenosis; ARAS, atherosclerotic renal artery stenosis; BMI, body mass index; SLDL-C, serum low density lipoprotein-cholesterol, S-HDL-C, serum high density lipoproteincholesterol; ns, not significant, NA, not applicable. Data are expressed as means \pm SD. * denotes $\mathrm{p}<0.05$ vs. healthy controls and $\dagger$ denotes $\mathrm{p}<0.05$ vs. non-RAS. P-values are for Kruskal-Wallis (K$\mathrm{W})$ non-parametric ANOVA. For categorical data chi square tests were performed

\section{Statistics}

Analyses were performed using one-way analysis of variance (ANOVA). If data were not normally distributed, Kruskal-Wallis one-way ANOVA on ranks was used. Unpaired t-test or Mann-Whitney U test was used when appropriate for comparisons between groups. Bonferroni corrections were made for multiple comparisons. To analyse within group effects of PTRA paired t-test or Wilcoxon's signed rank test was used for normally distributed and non-normally distributed data, respectively. The Pearson correlation (or Spearman correlation when data did not meet assumption about normality) coefficient was used to evaluate correlations. All tests were two-tailed and p-values $<0.05$ were considered significant. Results are presented as means \pm SD if not stated otherwise. Software SPSS 18.0.0 for windows (SPSS Inc., Chicago, USA) was used.

\section{Results}

\section{Patient characteristics at baseline}

Patient characteristics prior to PTRA are shown in table 1. There were no statistically significant differences between groups in age, BMI or gender distribution. Patients with ARAS more often had a diagnosis of diabetes. The prevalence of coronary artery disease and atrial fibrillation, and the duration of hypertension $(12 \pm 16$ vs. $12 \pm 15$ years in group ARAS and non-RAS, respectively) did not differ between groups. Plasma levels of serum LDL cholesterol (S-LDL-C) and HDL-cholesterol (S-HDL-C) were reduced in hypertensive groups vs. healthy controls (table 1 ). This finding could be explained by the fact that all hypertensive patients with suspected RAS were on statin treatment.

\section{Blood pressure, kidney function and biomarkers at baseline}

Ambulatory systolic and diastolic blood pressure and pulse pressure (PP) were significantly elevated in both hypertensive groups vs. healthy controls (table 2). In addition, ASBP and ambulatory PP were significantly elevated in group ARAS vs. the non-RAS group (table 2). There was no difference between hypertensive groups in the proportion of patients using diuretics or beta-blockers. Estimated GFR was reduced and urinary albumin excretion and serum creatinine levels elevated in both hypertensive groups vs. healthy controls (table 2 ). The blood leukocyte count differed between groups and was significantly elevated in the ARAS group vs. controls (table 2). There were no statistically significant differences between groups in serum levels of aldosterone, Ang II or in PRA (table 2). 


\section{Kidney \\ Blood Pressure Research}

Nowakowska-Fortuna/Saeed/Guron/Fu/Hammarsten/Jensen/Herlitz: Brain Natriuretic Peptides in Atherosclerotic Renal Artery Stenosis

Table 2. Blood pressure, kidney function and biomarkers at baseline

\begin{tabular}{lcccc}
\hline & $\begin{array}{c}\text { Healthy } \\
\text { controls } \\
(\mathrm{n}=20)\end{array}$ & $\begin{array}{c}\text { Non-RAS } \\
(\mathrm{n}=44)\end{array}$ & $\begin{array}{c}\text { ARAS } \\
(\mathrm{n}=47)\end{array}$ & $\begin{array}{c}\mathrm{p} \text {-value } \\
\mathrm{K} \text {-W test }\end{array}$ \\
\hline ASBP (mmHg) & $115 \pm 9$ & $140 \pm 16^{*}$ & $149 \pm 16^{*} \dagger$ & $\mathrm{p}<0.001$ \\
ADBP (mmHg) & $71 \pm 6$ & $81 \pm 9^{*}$ & $82 \pm 10^{*}$ & $\mathrm{P}<0.001$ \\
Ambulatory PP (mmHg) & $43 \pm 7$ & $59 \pm 16^{*}$ & $67 \pm 14^{*} \dagger$ & $\mathrm{P}<0.001$ \\
Antihypertensive drugs (n) & - & $2.6 \pm 1.1$ & $2.6 \pm 0.9$ & $\mathrm{NA}$ \\
Use of diuretics, $\mathrm{n}(\%)$ & - & $26(59 \%)$ & $33(70 \%)$ & $\mathrm{NA}$ \\
Use of beta-blockers, n (\%) & - & $39(87 \%)$ & $41(87 \%)$ & $\mathrm{NA}$ \\
S-creatinine ( $\mu$ mol/L) & $77 \pm 14$ & $115 \pm 42^{*}$ & $113 \pm 35^{*}$ & $\mathrm{p}<0.001$ \\
eGFR (ml/min/1.73m $\left.{ }^{2}\right)$ & $79 \pm 14$ & $56 \pm 20^{*}$ & $57 \pm 18^{*}$ & $\mathrm{p}<0.001$ \\
tU-albumin (mg/24hrs) & $7 \pm 4$ & $169 \pm 380^{*}$ & $97 \pm 175^{*}$ & $\mathrm{p}<0.01$ \\
B-WBC (x109/L) & $6.3 \pm 2.1$ & $7.1 \pm 2.2$ & $8.0 \pm 3.2^{*}$ & $\mathrm{p}<0.01$ \\
S-aldosterone (nmol/L) & $0.55 \pm 0.21$ & $0.56 \pm 0.40$ & $0.48 \pm 0.26$ & $\mathrm{~ns}$ \\
PRA (ng/ml/h) & $1.23 \pm 0.65$ & $1.71 \pm 2.4$ & $1.98 \pm 1.89$ & $\mathrm{~ns}$ \\
P-Ang II (pg/ml) & $12.0 \pm 4.9$ & $17 \pm 23.6$ & $13.2 \pm 8.8$ & $\mathrm{~ns}$ \\
S-BNP (ng/L) & $28 \pm 20$ & $48 \pm 68$ & $82 \pm 99^{*}$ & $\mathrm{p}<0.05$ \\
S-NT-proBNP (ng/L) & $87 \pm 55$ & $350 \pm 808^{*}$ & $521 \pm 737^{*}$ & $\mathrm{p}<0.001$ \\
P-adiponectin (mg/L) & $12.0 \pm 4.9$ & $10.3 \pm 6.2$ & $9.3 \pm 7.5^{*}$ & $\mathrm{p}<0.05$ \\
\hline PTRA; percutaneous transluminal renal angioplasty; RAS, renal artery stenosis; ARAS, & \\
atherosclerotic RAS; ASBP, ambulatory systolic blood pressure; ADBP, ambulatory diastolic blood \\
pressure; PP, pulse pressure; eGFR, estimated glomerular filtration rate according to the 4- \\
variable equation from the Modification of Diet in Renal Disease (MDRD) study; tU-albumin, total \\
24-h urinary albumin excretion; B-WBC, blood leukocyte count; PRA, plasma renin activity; P-Ang \\
II, plasma angiotensin II; BNP, brain natriuretic peptide; NT-proBNP, N-terminal pro-B-type \\
natriuretic peptide; ns, not significant, NA, not applicable. Data are expressed as means \pm SD. * \\
denotes p<0.05 vs. healthy controls and + denotes p<0.05 vs. non-RAS. P-values are for Kruskal- \\
Wallis (K-W) non-parametric ANOVA. For categorical data chi square tests were performed \\
\hline
\end{tabular}

\section{Brain natriuretic peptides and adiponectin at baseline}

Plasma BNP and NT-proBNP concentrations were elevated in patients with significant ARAS compared to healthy controls $(\mathrm{p}<0.05)$, but not in comparison to the non-RAS group (table 2). In addition, there was a statistically significant increase in NT-proBNP in the non-RAS group vs. controls $(\mathrm{p}<0.05)$. Plasma adiponectin was significantly reduced in the ARAS group compared to controls (table 2, $\mathrm{p}<0.05$ ), however, this difference did not reach statistical significance when patients with diabetes had been excluded from the analysis (data not shown).

In patients with ARAS, baseline BNP and NT-proBNP levels correlated significantly to age, ASBP, ambulatory PP, serum creatinine, eGFR and to urinary albumin excretion (table $3)$. Baseline BNP was also correlated to blood leukocyte count $(r=0.515 ; p<0.001)$. However, in multiple regression analysis only serum creatinine levels showed a statistically significant relationship to both BNP and NT-proBNP $(r=0.42, p<0.05$ for BNP; and $r=0.67, p<0.001$ for NT-proBNP). In multiple regression analysis baseline BNP was also significantly correlated to age $(\mathrm{p}<0.05)$, whereas NT-proBNP disclosed a relationship to ASBP $(\mathrm{p}<0.05)$.

Plasma adiponectin levels showed a significant correlation to BMI, ADBP and to S-HDL-C (table 3), but in multiple regression analysis only S-HDL-C showed a significant correlation $(\mathrm{p}<0.01$, figure 1). Notably, there was a statistically significant, and similar, correlation between plasma adiponectin and S-HDL-C also in the non-RAS group (figure 1).

\section{Effects of PTRA on blood pressure, kidney function and biomarkers}

Four weeks after PTRA, ASBP had decreased from $149 \pm 16$ to $139 \pm 15(\mathrm{p}<0.01$, figure 2$)$ and ADBP from $82 \pm 10$ to $77 \pm 11$ ( $\mathrm{p}<0.01$, figure 2 ). Also, ambulatory PP was reduced four weeks after PTRA (67 \pm 14 vs. $62 \pm 14 \mathrm{mmHg}$, before and after PTRA, respectively $\mathrm{p}<0.01$ ). The number of antihypertensive drugs was not significantly altered four weeks after PTRA (data not shown). Serum creatinine levels $(113 \pm 35$ vs. $112 \pm 37 \mu \mathrm{mol} / \mathrm{L}$, before and after PTRA, respectively, figure 2 ) and eGFR (data not shown) were not significantly affected by PTRA. 


\section{Kidney \\ Blood Pressure Research}

Nowakowska-Fortuna/Saeed/Guron/Fu/Hammarsten/Jensen/Herlitz: Brain Natriuretic Peptides in Atherosclerotic Renal Artery Stenosis

Table 3. Correlations between BNP, NT-proBNP, adiponectin and other variables at baseline in patients with significant atherosclerotic renal artery stenosis

\begin{tabular}{|c|c|c|c|}
\hline & S-BNP & S-NT-proBNP & P-Adiponectin \\
\hline Age (years) & $\mathrm{r}=0.506^{* *}$ & $\mathrm{r}=0.522^{* *}$ & ns \\
\hline BMI $\left(\mathrm{kg} / \mathrm{m}^{2}\right)$ & ns & ns & $r=-0.340 *$ \\
\hline ASBP (mmHg) & $\mathrm{r}=0.374^{* *}$ & $\mathrm{r}=0.520^{*}$ & ns \\
\hline ADBP (mmHg) & ns & ns & $r=0.308^{*}$ \\
\hline Ambulatory PP (mmHg) & $\mathrm{r}=0.515^{* *}$ & $r=0.640^{* *}$ & ns \\
\hline S-creatinine $(\mu \mathrm{mol} / \mathrm{L})$ & $\mathrm{r}=0.428^{* *}$ & $\mathrm{r}=0.476^{* *}$ & ns \\
\hline $\mathrm{eGFR}\left(\mathrm{ml} / \mathrm{min} / 1.73 \mathrm{~m}^{2}\right)$ & $\mathrm{r}=-0.547^{* *}$ & $\mathrm{r}=-0.644^{* *}$ & ns \\
\hline tU-albumin (mg/24hrs) & $r=0.327^{*}$ & $\mathrm{r}=0.365^{*}$ & ns \\
\hline S-HDL-C (mmol/L) & ns & ns & $\mathrm{r}=0.538^{*}$ \\
\hline
\end{tabular}

Statistically significant correlation coefficients are presented. BNP, brain natriuretic peptide;
NT-proBNP, N-terminal pro-B-type natriuretic peptide ARAS, atherosclerotic renal artery
stenosis; ASBP, ambulatory systolic blood pressure; ADBP, ambulatory diastolic blood
pressure; PP, pulse pressure; eGFR, estimated glomerular filtration rate according to the 4-
variable equation from the Modification of Diet in Renal Disease (MDRD) study; tU-Albumin,
total 24 -h urinary albumin excretion; S-HDL-C, serum high density lipoprotein-cholesterol. *
p $<0.05 ;{ }^{* *}$ p $<0.01$

Fig. 1. Relationship between plasma adiponectin concentration and serum levels of high-density lipoprotein (HDL) cholesterol in patients undergoing renal angiography because of suspected atherosclerotic renal artery stenosis (RAS). Out of these, 41 patients had no significant RAS (A) and 43 patients had atherosclerotic renal artery stenosis (ARAS) (B). There was a statistically significant correlation between plasma adiponectin levels and S-HDL cholesterol in both groups $(\mathrm{p}<0.01)$.

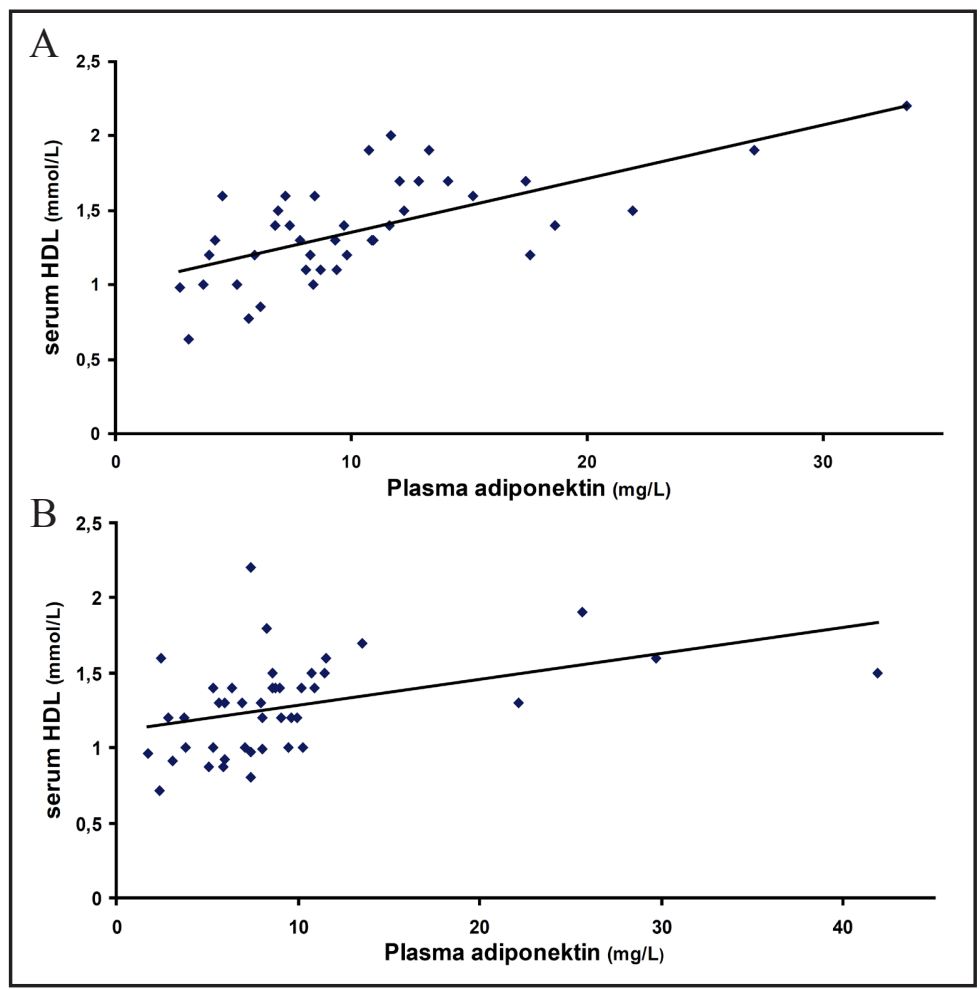

Serum aldosterone, plasma Ang II and PRA were not significantly affected by PTRA (data not shown). In addition, PTRA had no statistically significant effects on serum BNP $(82 \pm 99$ vs. $68 \pm 68 \mathrm{ng} / \mathrm{L}$, before and after PTRA, respectively, figure 3) or NT-proBNP (521 \pm 737 vs. $465 \pm 654 \mathrm{ng} / \mathrm{L}$, before and after PTRA, respectively, figure 3) when analysed four weeks after intervention.

Correlation of baseline data to changes in ASBP and BNP in response to PTRA

Changes in ASBP in response to PTRA were correlated to baseline ASBP ( $r=0.552$; $\mathrm{p}<0.001)$, ambulatory PP $(\mathrm{r}=0.380 ; \mathrm{p}<0.05)$, eGFR $(\mathrm{r}=-0.312$; $\mathrm{p}<0.05)$, plasma glucose $(\mathrm{r}=-$ 


\section{Kidney \\ Blood Pressure \\ Research}

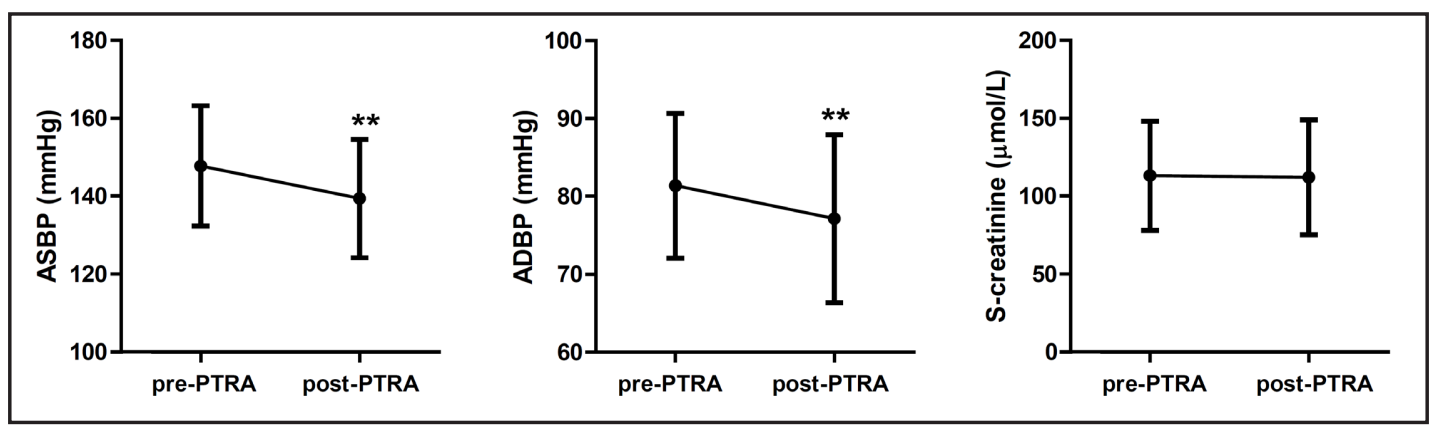

Fig. 2. Ambulatory systolic (ASBP) and diastolic (ADBP) blood pressure and serum creatinine levels before (pre-PTRA) and four weeks after (post-PTRA) percutaneous transluminal renal angioplasty (PTRA) in patients with atherosclerotic renal artery stenosis. Values are means \pm SD. ${ }^{* *}$ denotes $\mathrm{p}<0.01$ versus baseline.

Fig. 3. Serum concentrations of brain natriuretic peptide (BNP) and N-terminal pro-BNP (NTpro BNP) before (pre-PTRA) and four weeks after (post-PTRA) percutaneous transluminal renal angioplasty (PTRA) in patients with atherosclerotic renal artery stenosis. Values are means+SD. There were no statistically significant effects of PTRA.

Fig. 4. Correlation between change in ambulatory systolic blood pressure (SBP) and change in serum BNP concentration in response to transluminal renal angioplasty $(\mathrm{r}=0.57, \mathrm{P}<0.001)$ in patients with atherosclerotic renal artery stenosis.
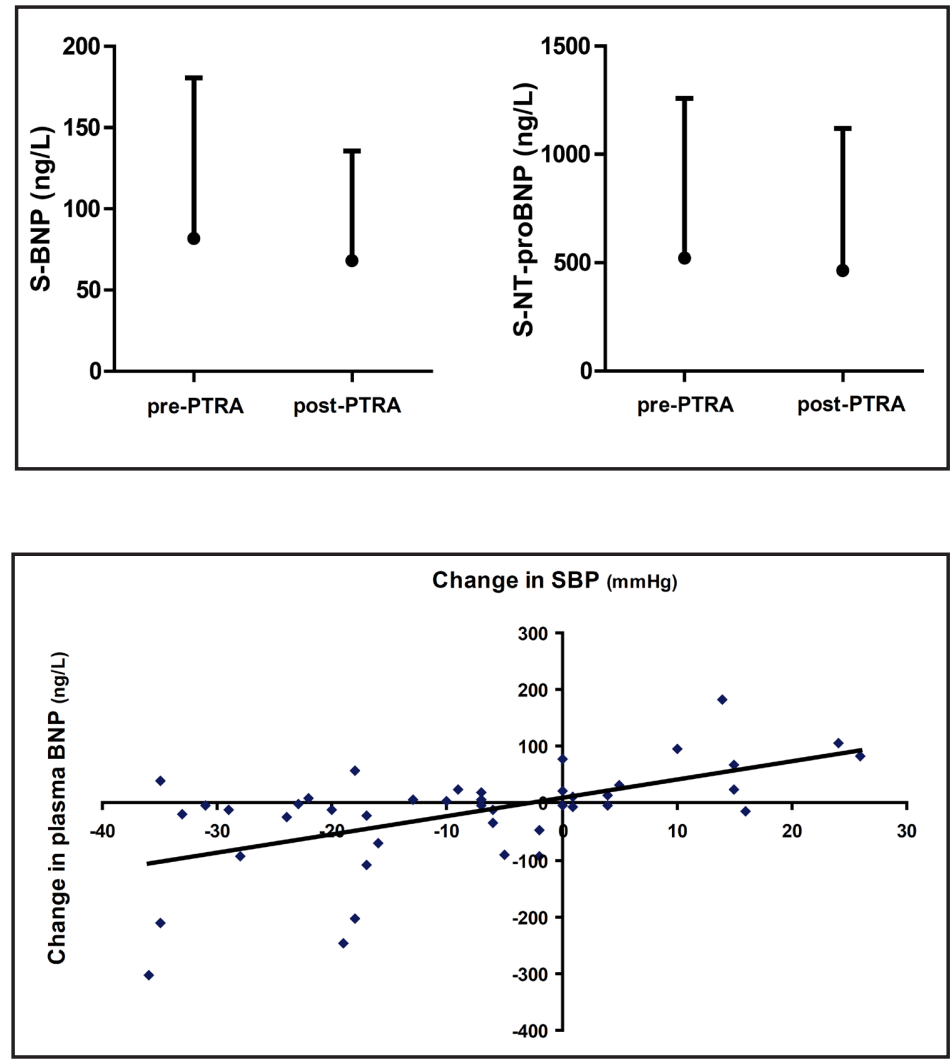

0.459; $\mathrm{p}<0.01)$, PRA ( $\mathrm{r}=0.306 ; \mathrm{p}<0.05)$ and to Ang II $(\mathrm{r}=0.474 ; \mathrm{p}<0.01)$. However, in a stepwise multiple regression analysis, only baseline ASBP, plasma glucose and Ang II significantly predicted changes in ASBP $(\mathrm{p}<0.05)$. The significant relationship to plasma glucose did not disappear when diabetic patients were excluded from the analysis.

There was no threshold plasma BNP concentration above which PTRA was shown to cause a more pronounced BP reduction. There was no significant difference in BP response to PTRA between patients from the highest and the lowest quartiles of baseline BNP values $(-13.3 \pm 5.3$ vs. $-7.6 \pm 3.9 \mathrm{mmHg}$ for ASBP and $-6.3 \pm 2.5$ vs. $-4.2 \pm 1.8 \mathrm{mmHg}$ for ADBP). However, there were statistically significant correlations between changes in ASBP and delta BNP $(r=0.57, p<0.001$, figure 4) and delta NT-proBNP $(r=0.57, p<0.001)$ in response to PTRA. 


\section{Kidney Blood Pressure Research}

Kidney Blood Press Res 2013;37:657-666

\begin{tabular}{l|l}
\hline DOI: $10.1159 / 000355746$ & (C) 2013 S. Karger AG, Basel
\end{tabular}

Published onlıne: December 15, 2013

www.karger.com/kbr

664

Nowakowska-Fortuna/Saeed/Guron/Fu/Hammarsten/Jensen/Herlitz: Brain Natriuretic

Peptides in Atherosclerotic Renal Artery Stenosis

\section{Discussion}

The main results of the present study were that hypertensive patients with ARAS showed significantly elevated plasma concentrations of BNP and NT-proBNP, but reduced adiponectin levels, compared to healthy controls. However, concentrations of these biomarkers were not significantly different in ARAS patients versus hypertensive individuals without RAS. In addition, although PTRA did not significantly affect BNP or NT-proBNP concentrations in patients with ARAS, there was a significant correlation between changes in plasma BNPs and changes in blood pressure in response to intervention.

Our observation that plasma concentrations of BNPs were elevated in ARAS patients vs. healthy controls, but not compared to hypertensive patients without RAS, clearly suggest that other causes than RAS per se were responsible for the increased BNP levels in ARAS patients. In fact, multiple regression analysis showed that serum creatinine was the only variable that was significantly correlated to both plasma BNP and NT-proBNP levels in patients with ARAS. Hence, differences in kidney function likely explained the increase in BNP and NT-proBNP in ARAS subjects vs. healthy controls since ARAS patients had significantly elevated serum creatinine levels reflecting reduced GFR. On the contrary, serum creatinine concentrations were similar in hypertensive patients with or without RAS. Notably, in our study baseline levels of plasma BNP and NT-proBNP could not be used to predict the outcome of PTRA on blood pressure and we could not identify a pre-interventional plasma BNP or NTproBNP level above which a successful revascularization could be expected. Still, there was a significant correlation between changes in plasma BNPs and changes in blood pressure in response to PTRA. The mechanisms underlying this correlation remain speculative but could theoretically be explained by reduced left ventricular afterload secondary to the fall in blood pressure, decreased activity of the RAAS, or increased natriuresis following restoration of pressure-natriuresis in the previously stenotic kidney.

Our data do not confirm the results of two previous publications in which baseline BNP levels were shown to predict the blood pressure response after revascularization in patients with RAS $[18,19]$. Silva et al [18] showed that BNP was increased in 27 patients with severe ARAS, and had decreased the day after successful stent revascularization. In addition, a baseline BNP level $>80 \mathrm{pg} / \mathrm{ml}$ appeared to be a good predictor of a favourable blood pressure response to revascularization. Staub et el [19] showed in 120 hypertensive patients with RAS that BNP levels were elevated, and decreased after revascularization, and concluded that a pre-interventional BNP $>50 \mathrm{pg} / \mathrm{ml}$ may be helpful to identify patients in whom PTRA will improve blood pressure. There are a number of possible explanations for the discrepant results in our study compared to those previously published. First, in both the earlier studies $[18,19]$ patients with bilateral RAS were included whereas we only investigated patients with unilateral RAS. In the study by Silva et al [18] the proportion of patients with bilateral RAS was as high as $33 \%$. It is feasible to speculate that patients with bilateral RAS, who in general have a more volume-dependent hypertension, would have increased plasma levels of BNP at baseline and respond to revascularization with a more prominent BNP reduction. Secondly, Staub et al [19] included patients with fibromuscular dysplasia, who made up $22 \%$ of all patients with a favourable blood pressure response, while only patients with ARAS participated in the present study. Thirdly, the majority of patients included in the two earlier studies were on on-going treatment with ACE inhibitors or ARBs whereas none of the patients in our study were on medications that inhibited the RAAS. It is well known that cardiac BNP synthesis and release is stimulated by both hemodynamic mechanisms and by direct, non-hemodynamic, pathways via neuroendocrine factors such as Ang II $[7,8]$. In our study, PTRA had no significant effects on either plasma Ang II levels or PRA and it is therefore possible that Ang II-driven BNP production prevented reductions in plasma BNP levels following intervention.

Interestingly, Chrysochou et al [20] investigated the prognostic role of NT-proBNP in patients with ARAS and found that raised NT-proBNP levels were associated with a greater likelihood of death when subdivided by CKD stage. Thus, although these authors showed 


\section{Kidney \\ Blood Pressure Research}

Kidney Blood Press Res 2013;37:657-666

\begin{tabular}{l|l}
\hline DOI: $10.1159 / 000355746$ & C 2013 S. Karger AG, Basel
\end{tabular}

Published onlıne: December 15, 2013

www.karger.com/kbr

Peptides in Atherosclerotic Renal Artery Stenosis

that kidney function was a more important risk factor for death there was an additive risk effect of NT-proBNP. Hence, measurements of BNPs might be helpful in identifying ARAS patients with increased risk and in need of intensified management.

The significant correlation between low plasma adiponectin levels and low S-HDL-C concentrations that we observed in both hypertensive groups (ARAS and non-RAS) in the present study is compatible with previous findings in patients with the metabolic syndrome and in type 2 diabetes [21]. It has previously been shown that low adiponectin levels predict elevated plasma VLDL-apoB concentrations through impaired catabolism of VLDL-apoB [22]. Adiponectin could therefore influence HDL metabolism by regulating the metabolism of triglyceride-rich lipoproteins [22]. Hence, it is possible that suppressed adiponectin levels in ARAS patients may contribute to the dyslipidaemia previously described in this patient group [17].

\section{Conclusion}

Hypertensive patients with ARAS have elevated plasma concentrations of BNP and NT-proBNP, but reduced adiponectin levels, compared to healthy controls. However, concentrations of these biomarkers were not significantly different in ARAS patients versus hypertensive individuals without RAS, and the increases in plasma BNP and NT-proBNP vs. healthy controls were most likely explained by a reduced kidney function in ARAS patients. In addition, baseline levels of BNPs could not be used to predict the outcome of PTRA on blood pressure.

\section{Conflict of interest statement}

The results presented in this paper have not been published previously in whole or part, except in abstract format.

\section{Acknowledgements}

This study was supported by grants from the Swedish Heart-Lung Foundation, the Swedish state under the LUA/ALF agreement, the Göteborg Medical Society, the Swedish Medical Society, the Swedish Association for Kidney Patients, the Swedish Society of Nephrology, Britt Wennerström's Research Foundation, Inger Bendix Foundation, Paul Frankenius Foundation, AstraZeneca, Mölndal, Sweden, IngaBritt and Arne Lundbergs Research Foundation, and Marianne and Marcus Wallenbergs Foundation. We thank Elisabeth Ericson, Inger Olander, Lisbeth Selven, and Lotta Sundström for expert technical assistance.

\section{References}

\footnotetext{
1 Mukoyama M, Nakao K, Hosoda K, Suga S, Saito Y, Ogawa Y, Shirakami G, Jougasaki M, Obata K, Yasue $\mathrm{H}$, Kambayashi Y, Inouye K, Imura H: Brain natriuretic peptide as a novel cardiac hormone in humans: evidence for an exquisite dual natriuretic peptide system, atrial natriuretic peptide and brain natriuretic peptide. J Clin Invest 1991;87:1402-1412.

2 Morrison LK, Harrison A, Krishnaswamy P, Kazanegra R, Clopton P, Maisel A: Utility of a rapid B-natriuretic peptide assay in differentiating congestive heart failure from lung disease in patients with dyspnea. J Am Coll Cardiol 2002;39:202-209.
} 


\section{Kidney \\ Blood Pressure Research}

Nowakowska-Fortuna/Saeed/Guron/Fu/Hammarsten/Jensen/Herlitz: Brain Natriuretic Peptides in Atherosclerotic Renal Artery Stenosis

-3 Troughton RW, Frampton CM, Yandle TG, Espiner EA, Nicholls MG, Richards AM: Treatment of heart failure guided by plasma aminoterminal brain natriuretic peptide (N-BNP) concentrations. Lancet 2000;355:126130.

4 Mair J: Biochemistry of B-type natriuretic peptide--where are we now? Clin Chem Lab Med 2008;46:15071514.

5 Bionda C, Bergerot C, Ardail D, Rodriquez-Lafrasse C, Rousson R: Plasma BNP and NT-proBNP assays by automated immunoanalyzers: analytical and clinical study. Ann Clin Lab Sci 2006;36:299-306.

6 Vickery S, Price CP, John RI, Abbas NA, Webb MC, Kempson ME, Lamb EJ: B-type natriuretic peptide (BNP) and amino-terminal proBNP in patients with CKD: relationship to renal function and left ventricular hypertrophy. Am J Kidney Dis 2005;46:610-620.

7 McGrath MF, de Bold ML, de Bold AJ: The endocrine function of the heart. Trends Endocrinol Metab 2005;16:469-477.

8 Wiese S, Breyer T, Dragu A, Wakili R, Burkard T, Schmidt-Schweda S, Füchtbauer EM, Dohrmann U, Beyersdorf F, Radicke D, Holubarsch CJ: Gene expression of brain natriuretic peptide in isolated atrial and ventricular myocardium: Influence of angiotensin II and diastolic fiber length. Circulation 2000;102:30743079.

9 Martinez-Maldonado M: Pathophysiology of renovascular hypertension. Hypertension 1991;17:707-719.

10 Nishimura M, Milsted A, Bloch CH, Brosnihan KB, Ferrario CM: Tissue renin-angiotensin systems in renal hypertension. Hypertension 1992;20:158-167.

11 Fortuño A, Rodríguez A, Gómez-Ambrosi J, Frühbeck G, Díez J: Adipose tissue as an endocrine organ: role of leptin and adiponectin in the pathogenesis of cardiovascular diseases. J Physiol Biochem 2003;59:51-60.

12 Maeda K, Okubo K, Shimomura I, Funahashi T, Matsuzawa Y, Matsubara K: cDNA cloning and expression of a novel adipose-specific collagen-like factor, apMI (adipose most abundant gene transcript I). Biochem Biophys Res Commun 1996;221:286-289.

13 Matsuzawa Y: Adiponectin: Identification, physiology and clinical relevance in metabolic and vascular disease. Atheroscler Suppl 2005;6:7-14.

14 Saeed A, Herlitz H, Nowakowska-Fortuna E, Nilsson U, Alhadad A, Jensen G, Mattiasson I, Lindblad B, Gottsäter A, Guron G: Oxidative stress and endothelin-1 in atherosclerotic renal artery stenosis and effects of renal angioplasty. Kidney Blood Press Res 2011;34:396-403.

-15 Nowakowska Fortuna E, Herlitz H, Saeed A, Attman PO, Jensen G, Alaupovic P, Guron G: Lipoprotein abnormalities in patients with atherosclerotic renovascular disease. Kidney Blood Press Res 2011;34:311319.

16 Tunstall-Pedoe H, Kuulasmaa K, Amouyel P, Arveiler D, Rajakangas AM, Pajak A: Myocardial infarction and coronary deaths in the World Health Organization MONICA Project. Registration procedures, event rates, and case-fatality rates in 38 populations from 21 countries in four continents. Circulation 1994;90:583612.

17 Alhadad A, Guron G, Fortuna-Nowakowska E, Saeed A, Mattiasson I, Jensen G, Lindblad B, Gottsäter A, Herlitz H: Renal angioplasty causes a rapid transient increase in inflammatory biomarkers, but reduced levels of interleukin-6 and endothelin-1 1 month after intervention. J Hypertens 2007;25:1907-1914.

18 Silva JA, Chan AW, White CJ, Collins TJ, Jenkins JS, Reilly JP, Ramee SR: Elevated brain natriuretic peptide predicts blood pressure response after stent revascularization in patients with renal artery stenosis. Circulation 2005;111:328-333.

19 Staub D, Zeller T, Trenk D, Maushart C, Uthoff H, Breidthardt T, Klima T, Aschwanden M, Socrates T, Arenja N, Twerenbold R, Rastan A, Sixt S, Jacob AL, Jaeger KA, Mueller C: Use of B-type natriuretic peptide to predict blood pressure improvement after percutaneous revascularisation for renal artery stenosis. End J Vasc Endovasc Surg 2010;40;599-607.

20 Chrysochou C, Manzoor S, Wright J, Roberts SA, Wood G, McDowell G, Kalra PA: Role of renal function and cardiac biomarkers (NT-proBNP and troponin) in determining mortality and cardiac outcome in atheromatous renovascular disease. Kidney Blood Press Res 2009;32:373-379.

21 Matsuzawa Y, Funahashi T, Kihara S, Shimomura I: Adiponectin and metabolic syndrome. Arterioscler Thromb Vasc Biol 2004;24:29-33.

22 Ng TW, Watts GF, Farvid MS, Chan DC, Barrett PH: Adipocytokines and VLDL metabolism: independent regulatory effects of adiponectin, insulin resistance, and fat compartments on VLDL apolipoprotein B-100 kinetics? Diabetes 2005;54:795-802. 